\title{
The Nuclear Physics Program at the NESR Storage Ring at FAIR
}

\section{Nasser Kalantar-Nayestanaki ${ }^{* *}$}

KVI, University of Groningen

Zernikelaan 25, Groningen, 9747 AA, The Netherlands

E-mail: nasserakvi.nl

In this contribution, the physics program for the experiments at the New Experimental Storage Ring (NESR) at FAIR, namely ILIMA, AIC, ELISe and EXL, will be briefly outlined. All these experiments aim to study the structure and the dynamics of radioactive ions as they circulate in a ring, or collide with either light ions in an inverse kinematics, or electrons and anti-protons in a collider mode. Although some of the physics issues addressed in all four are rather similar, the experimental equipment used for them are quite different. The experimental setup for EXL will also be briefly discussed in this contribution.

8th International Conference on Nuclear Physics at Storage Rings-Stori11,

October 9-14, 2011

Laboratori Nazionali di Frascati dell'INFN, Italy

\footnotetext{
${ }^{*}$ Speaker.

${ }^{\dagger}$ For the ILIMA, AIC, ELISe and EXL collaborations
} 


\section{Introduction}

With the advent of new facilities producing high-intensity radioactive ion beams around the world, the field of nuclear structure has received a tremendous boost. Many activities have been initiated to capitalize on various aspects of the new facilities. The Facility for Anti-proton and Ion Research (FAIR) in Darmstadt, Germany is one of these facilities which is capable of producing beams of nuclei far from the valley of stability. These beams will be distributed among various experimental setups which are used in different subfields ranging from high-precision spectroscopy to reactions in nuclei under extreme conditions such as very large neutron-proton asymmetry [1].

For the direct measurement of masses and lifetimes of radioactive species, it is best to circulate the ions in a ring and study their properties. In the reaction studies, since the nucleus under investigation is the one which is produced in the process of in-flight fragmentation, one has to deal with inverse kinematics in which the hadronic probe, generally a light nucleus, is the target being bombarded by the heavy (radioactive) nucleus. The inverse kinematics will impose particular conditions on the design of detection systems. In order to take full advantage of the new FAIR facility, the plan is to have a storage ring in which four different experimental programs will be pursued. These are the ILIMA [2] project in the ring, an internal target hall (EXL, EXotic nuclei studied in Light-ion induced reactions [3]), an electron-ion collider (ELISe, ELectron-Ion Scattering experiment in a storage ring [4]) and an Anti-proton Ion Collider, (AIC) [5]. All these programs deal with the bulk properties (such as masses, lifetimes, matter and charge radii) of nuclei, giant resonances, spectroscopic information on valence nucleons, and reaction mechanisms governing the nuclei. ILIMA will measure directly the masses and the lifetimes of radioactive ions. EXL will specialize in low-momentum-transfer aspects of nuclear structure. AIC will be specifically suited for measurements of proton and neutron rms radii separately and ELISe will investigate nuclear properties with an electromagnetic probe. The beam energy used in these studies generally ranges from $200 \mathrm{MeV} /$ nucleon to $1 \mathrm{GeV} /$ nucleon. For the case of the electron collider, electron beam energies from $125 \mathrm{MeV}$ to $500 \mathrm{MeV}$ will be used, yielding energies of up to $1.5 \mathrm{GeV}$ in the center of mass. Anti-protons will have an energy of $30 \mathrm{MeV}$ for the collision with radioactive ions. Figure 1 shows part of the proposed FAIR facility primarily used for nuclear-structure studies. EXL, ELISe and AIC are located in NESR while ILIMA experiments can be done in both NESR and $\mathrm{CR}$ depending on the lifetime of the radioactive beam. In the following more details will be given on the physics case for two of the setups, namely EXL and ELISe. For the details of the ILIMA program, see Ref. [6] and for the techniques used for the in-ring mass and lifetime measurements, see elsewhere in these proceedings. The case for AIC can be found in more details in Ref. [7, 8].

\section{Physics and experimental setup of EXL}

In the study of the structure of nuclei, one has to perform measurements in a large range of momentum transfers in order to fully understand the details. However, the experimental conditions for low-momentum and high-momentum-transfer measurements are quite different. Since all studies of interest will be carried out in inverse kinematics, a low-momentum-transfer measurement necessitates the utilization of storage rings due to very small recoil energies. These low-energy particles would simply be stopped in any external target. The alternative would be to use active targets 


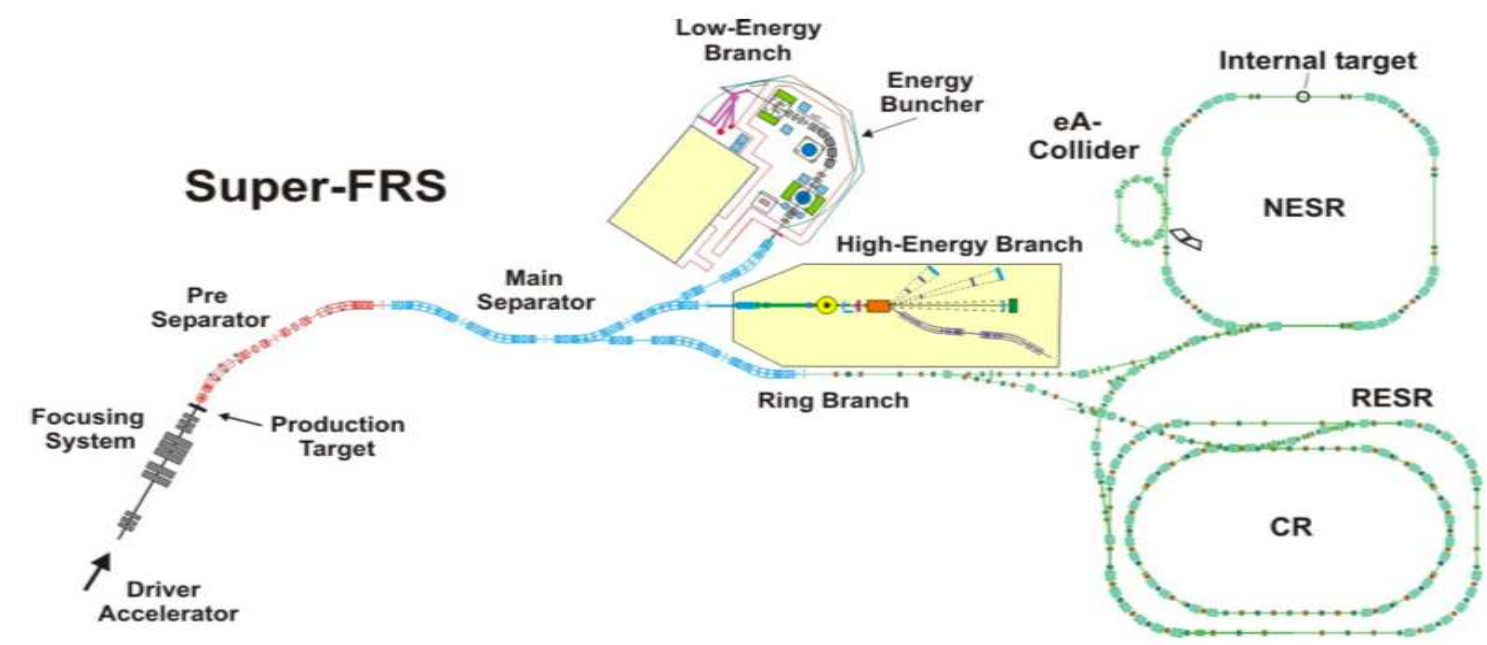

Figure 1: The overview of the beam lines after the main part of the Super-FRS leading to NuSTAR experiments. EXL, ELISe and AIC are placed in the NESR ring while ILIMA is located both in the NESR as well as the CR.

but that will only be suitable for low luminosities. One should then perform these measurements in internal-target facilities in which the target thickness must be very small to allow a reasonable life-time for the beam. The very small target thicknesses used in the rings are compensated for by circulating the beam in the ring a couple of million times a second increasing, thereby, the effective luminosity, making the in-ring experiments comparable to external-target measurements as far as the luminosity is concerned. In addition, the large momentum spread and the large emittance of the beam coming from the Super-FRS should be reduced in order to achieve the goals of the lowmomentum-transfer measurements. This is done by cooling the beam in the storage ring. One clear advantage of performing the experiments in the storage ring is the lack of background from the target windows which are present in the external-target experiments. The high-momentum-transfer measurements, on the other hand, do not suffer from the above-mentioned energy limitations and as such are better carried out in external-target facilities. The intensity of the beams delivered from Super-FRS could in fact be low, since the required luminosity can be obtained by making the target thicker. To this end, the compromise between the energies of the outgoing particles, their required resolutions and the cross sections of a given reaction determines what target thickness one can use. In short, one would need a storage ring to perform the low-momentum-transfer measurements and an external target for the high-momentum-transfer measurements. The EXL and $\mathrm{R}^{3} \mathrm{~B}$ (placed in the high-energy branch shown in Fig. 1) experiments are designed to address these two aspects and are, in that sense, completely complementary to each other.

The key physics issues being covered with the EXL (and $\mathrm{R}^{3} \mathrm{~B}$ for the high-momentum transfer measurements) program are:

- nuclear matter distributions near the drip lines. The halo structures as well as the nuclear skins will be thoroughly studied;

- the isospin-dependence of the single-particle shell structures (new magic numbers, new shell 
gaps and spectroscopic factors);

- nucleon-nucleon correlations and cluster formation;

- new collective modes (different deformations for protons and neutrons), giant and pygmy resonances with different multipolarities;

- in-medium interactions in asymmetric and low-density nuclear matter;

- parameters of nuclear equation of state;

- the astrophysical r- and rp-processes in the form of Gamow-Teller transitions, neutron capture, etc..

All these can be done in the light-ion induced direct reactions, such as elastic $(p, p),(\alpha, \alpha)$ scattering, inelastic scattering in $\left(p, p^{\prime}\right),\left(\alpha, \alpha^{\prime}\right)$, charge-exchange reactions of the type $(p, n)$ or $\left({ }^{3} \mathrm{He}, t\right)$, and $\left(d,{ }^{2} \mathrm{He}\right)$, quasi-free scattering like $(p, 2 p),(p, p n)$, and $(p, p+c l u s t e r)$ and finally the transfer reactions of the type $(p, t),\left(p,{ }^{3} \mathrm{He}\right),(p, d)$, and $(d, p)$. The relevant information should be extracted from an energy and momentum measurement of the outgoing particles. In these experiments, it should be possible to detect many of the outgoing particles, making most of the channels kinematically (over)complete. This is particularly necessary in reducing the backgrounds in measurements when the cross sections are very low. An example of the type of measurements which could be performed with EXL is shown in Fig. 2 [9]. With elastic proton scattering on radioactive isotope and, in particular, at low momentum transfers, one can deduce the matter-density distribution. This, combined with electron scattering on the same object (a la ELISe) will result in neutron density distributions.
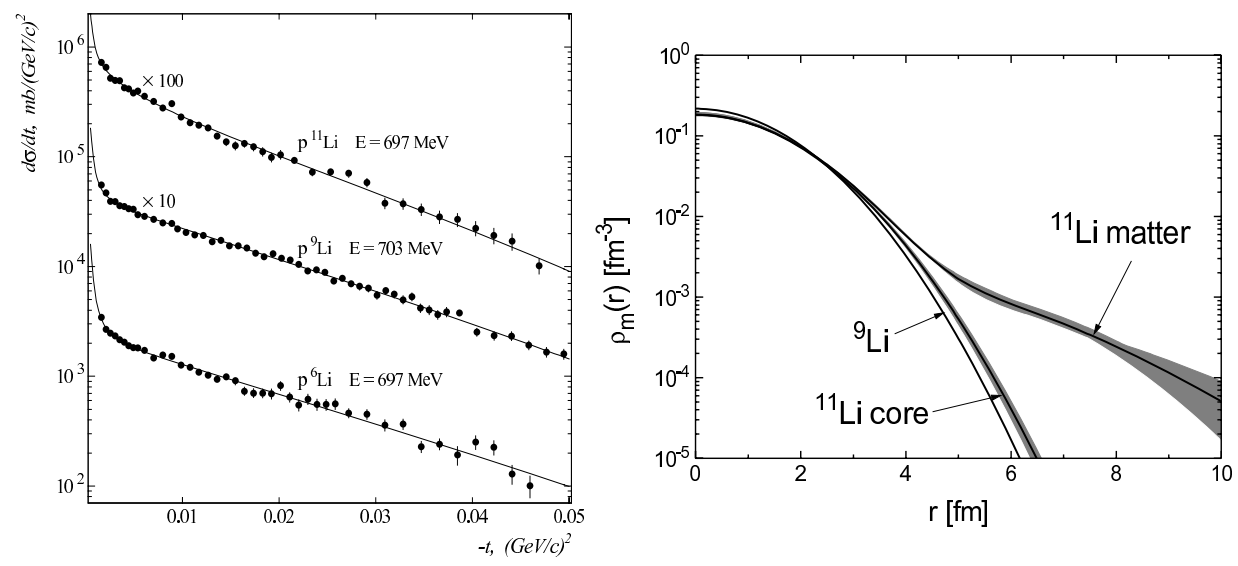

Figure 2: Elastic proton scattering from Li ions. Cross sections are shown on the left, while the resulting densities are depicted on the right. Here, the halo structure of ${ }^{11} \mathrm{Li}$ is quite nicely observed.

\subsection{EXL setup}

Within the EXL Technical Proposal, the design of a complex detection system was investigated with the aim to provide a high-efficiency, high-resolution, and a universal setup, applicable to a 


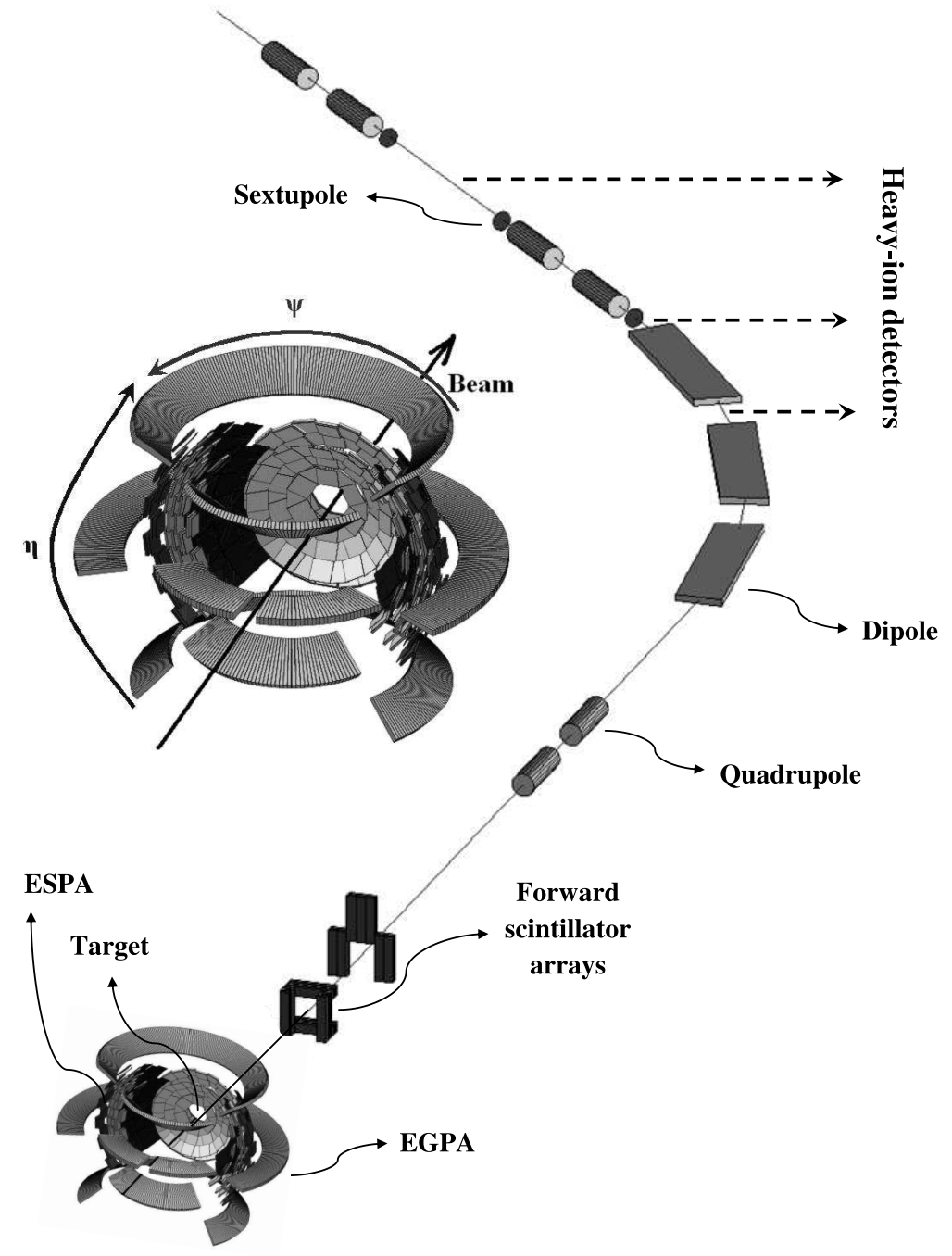

Figure 3: The overview of the EXL setup with various components shown. The recoil detector is enlarged and shown in the middle. Note that only a few rings of the calorimeter and some segments of the Si array are drawn here for the sake of clarity.

wide class of reactions (see Fig. 3). The apparatus is foreseen to be installed at the internal target of the NESR storage cooler ring. The detection system includes:

- a Si-strip and $\mathrm{Si}(\mathrm{Li})$ detector array for recoiling target-like reaction products, completed by slow-neutron detectors (not shown in the figure), and a calorimeter with a high granularity for $\gamma$-rays and for the total-energy measurement of more energetic target recoils;

- detectors in forward direction (schematically shown in the figure as forward scintillator arrays) for fast ejectiles from the excited projectiles, i.e. neutrons and light charged particles;

- heavy-ion detectors for the detection of beam-like reaction products. 
All detector components will practically cover the full relevant solid angle and have detection efficiencies close to unity. With this setup, kinematically-complete measurements will become possible.

Major research and development work is required for the design and the technical implementation of the target-recoil detector which will be the most challenging part of the project. In particular, the detector components need to fulfil strong demands concerning angular and energy resolutions, detection thresholds, dynamic range, granularity, vacuum compatibility, etc., partly not available or derivable from the existing detection technologies. A second major task is to achieve high densities in the internal gas-jet target, of the order of $10^{14}-10^{15}$ atoms $/ \mathrm{cm}^{2}$ or above with a well localized interaction zone [10]. On both fronts, there are major developments going on with very promising results. In 2005 and 2011, feasibility measurements in the present ESR ring at GSI were performed with a small number of detectors representing various components of the EXL detection system $[11,12,13,14,15]$. These included a Si-strip detector placed in vacuum, plastic scintillators to detect the fast forward recoil particles, and a combination of a p-i-n diode and a scintillator placed after the first bending magnet to detect the projectile-like heavy ions. The results of both measurements are very promising and pave the way for the final design of the setup. Prototyping of various components, and specially those which have to operate under the ultra-high vacuum condition are well underway $[16,17]$ (see the contribution of Streicher et al. elsewhere in these proceedings.).

\section{Physics case of ELISe}

The advantage of using electrons instead of hadronic probes is two-fold. Electrons are pointlike objects and the interaction of electrons with matter is very well understood in the framework of QED. In addition, the interaction is weak, making it possible to have a very good description of the collision process already in the first order of the perturbation theory. The second advantage of electron scattering is that, with the exchange of photon, the process is space-like allowing one to fix the excitation energy of any state and study the interaction for that particular state as a function of momentum transfer. These advantages have been used in the past to study, for example, the charge distribution of nuclei [18]. These studies can now be extended to exotic nuclear matter. The (new) collective states and giant resonances which were described in the EXL section can also be well studied with electron scattering with its high selectivity to different multipolarities. Furthermore, electro-fission can be employed with this facility due to the fact that the outgoing fissioning particles will be primarily going in the direction of the primary beam, however, with different rigidities allowing them to be easily detected. Depending on the luminosity of the beam, one can go further and study quasi-free scattering, such as $\left(e, e^{\prime} N\right)$ or even cluster knock-out, to provide information on the internal structure of the nucleus and to study nucleon-nucleon correlations in nuclear medium [19].

Here, I will give one example of the power of electron scattering as studied with ELISe. In this example, an experiment is simulated the results of which are shown in Fig. 4. For simplicity, two known cases have been taken for this study, namely ${ }^{12} \mathrm{C}$ and ${ }^{208} \mathrm{~Pb}$. A center of mass energy of $400 \mathrm{MeV}$, a heavy-ion beam energy of $740 \mathrm{AMeV}$, a luminosity of $10^{28} \mathrm{~cm}^{-2} \mathrm{~s}^{-1}$, and a detection solid angle of $100 \mathrm{msr}$ are assumed for these simulations. In addition, we have assumed that the spectrometer has an opening angle of $20^{\circ}$ which results in a simultaneous measurement of the 
points within the vertical bars. These "data points" would then be obtained in a run of four weeks. One can see that the Fourier transformation of these "measured" points results in rather precise charge-density distributions which, in turn, can be compared with theoretical predictions shown for ${ }^{208} \mathrm{~Pb}$ in the picture. The error bands, observed in the picture, are the results of the limited q-range of the measurements and the statistical accuracy of the points measured. To obtain these results, we started from the known charge-density distributions, Fourier transformed them to calculate the expected cross sections and made a Monte-Carlo randomization around these expected values.
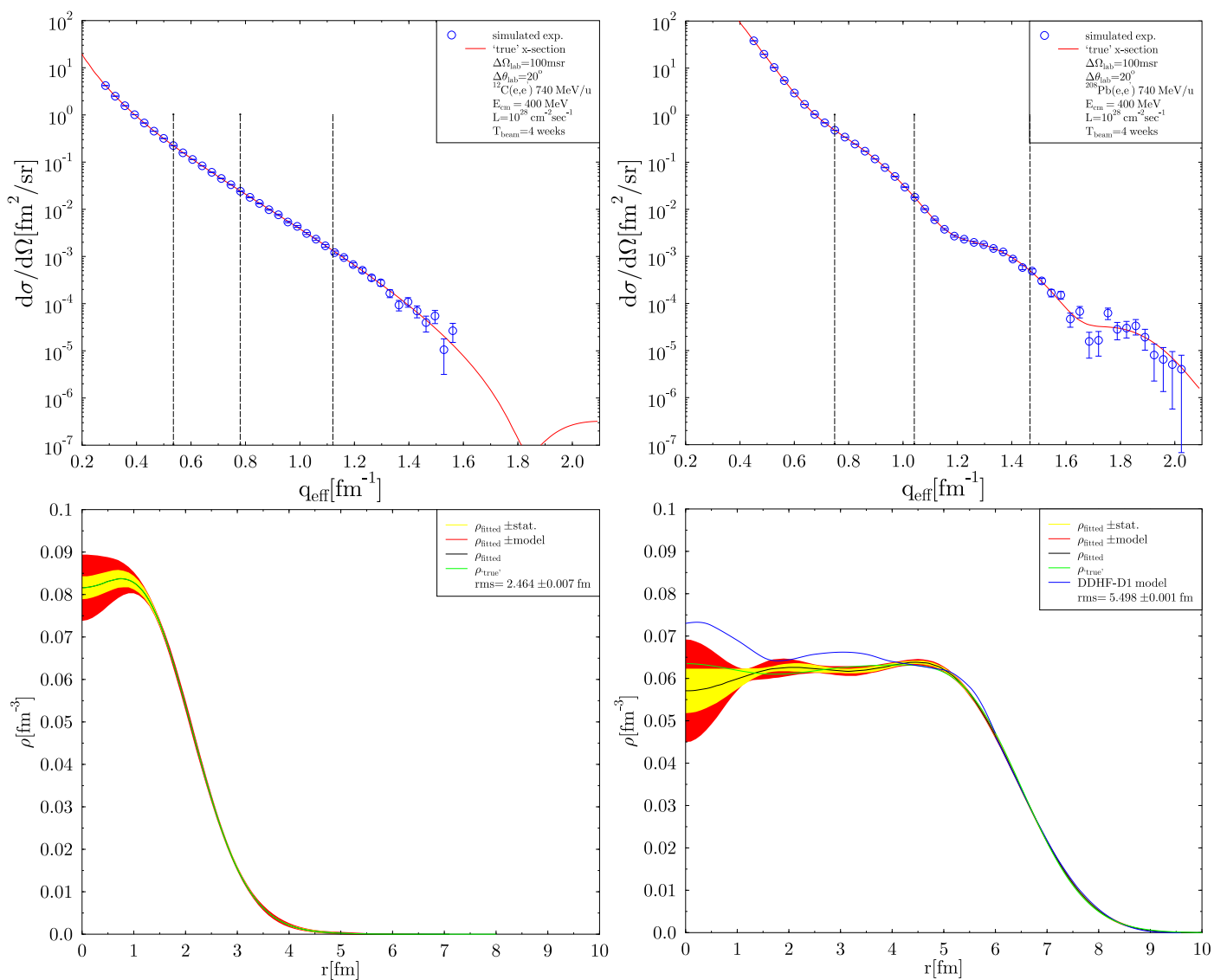

Figure 4: Results of the simulations for elastic electron scattering from ${ }^{12} \mathrm{C}$ (left) and ${ }^{208} \mathrm{~Pb}$ (right). The curve shown in the lower-right panel is the result of a mean-field calculation for the charge-density distribution.

The spectrometer which has to perform the task of detecting the outgoing electrons has to be a very special one. First, one has to guide the scattered electrons out of the beam environment without disturbing the circulating beams of electrons and heavy ions. Subsequently, these electrons should be momentum analyzed in a second magnet with special detectors at its focal plane. For more details of the design of this magnet, see the contribution of Simon et al. elsewhere in these proceedings and Refs. [20, 21]. 


\section{Conclusions}

In this contribution, the physics case for three of the NuSTAR experiments, EXL and ELISe and very briefly AIC and ILIMA were discussed and the experimental setup for EXL was also introduced. Presently, these collaborations are performing intense R\&D to finalize their designs. Their Technical Design Reports are expected within the next year or two. On the way to the final experiments, several measurements have been done or are planned to be performed with the present facility at GSI albeit with down-graded specifications as compared with the final design. The first measurements with beams from the Super-FRS are expected in 2017 in the high-energy beam-line.

\section{ACKNOWLEDGEMENT}

I would like to thank all my colleagues and collaborators from the ILIMA, AIC, ELISe, and EXL collaborations.

\section{References}

[1] http://www.gsi.de/fair/experiments/NUSTAR/projects_e.html.

[2] http://www.gsi.de/forschung/fair_experiments/NUSTAR/ilima_e.html.

[3] http://www.rug.nl/kvi/Research/hnp/Research/EXL/index?lang=en.

[4] http://www.gsi.de/fair/experiments/elise/index_e.html.

[5] http://www.gsi.de/forschung/fair_experiments/NUSTAR/AIC_e.html.

[6] Y.A. Litvinov et al., Proc. of the $11^{\text {th }}$ Symposium on Nuclei in the Cosmos, Heidelberg, Germany, July 2010, PoS (NIC XI) 073.

[7] P. Kienle, Nucl. Instr. Meth. B 214, 193 (2004).

[8] H. Lenske and P. Kienle, Phys. Lett. B647, 82 (2007).

[9] P. Egelhof et al., Eur. Phys. J. A 15, 27 (2002).

[10] M. Kühnel et al., Nucl. Inst. and Meth. in Phys. Res. A602, 311 (2009).

[11] S. Ilieva, Ph.D. Thesis, University of Mainz, 2009.

[12] H. Moeini, Ph.D. Thesis, University of Groningen, 2010.

[13] S. Ilieva et al., Eur. Phys. J. ST 150, 357 (2007).

[14] N. Kalantar-Nayestanaki et al., Int. Jour. of Mod. Phys. E 18, No. 2, 524 (2009).

[15] H. Moeini, S. Ilieva et al., Nucl. Inst. and Meth. in Phys. Res. A634, 77 (2011).

[16] M. von Schmid et al., Nucl. Inst. and Meth. in Phys. Res. A629, 197 (2011).

[17] B. Streicher et al., Nucl. Inst. and Meth. in Phys. Res. A654, 604 (2011).

[18] H. de Vries, C.W. de Jager, C. de Vries, At. Data Nucl. Data Tables 36, 495 (1987).

[19] A.N. Antonov et al., Nucl. Inst. and Meth. in Phys. Res. A637, 60 (2011).

[20] G.P.A. Berg et al., Nucl. Inst. and Meth. in Phys. Res. A640, 123 (2011).

[21] T. Adachi et al., Nucl. Inst. and Meth. in Phys. Res. A659, 198 (2011). 